\title{
Paint-like Compositing Based on RYB Color Model
}

\author{
Junichi Sugita* \\ Tokyo Healthcare University
}

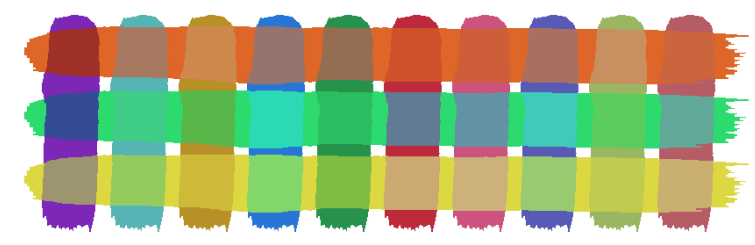

(a) $\alpha$-compositing $(\alpha=0.6)$
Tokiichiro Takahashi* ${ }^{*}$

Tokyo Denki University / UEI Research

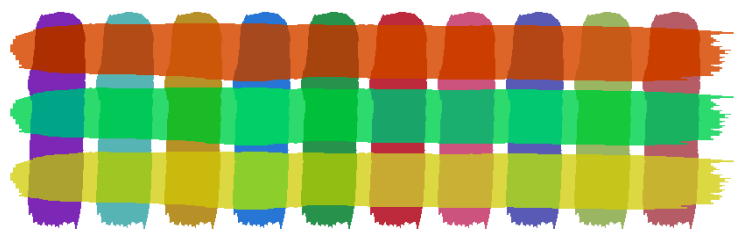

(b) Modified $\alpha$-compositing ( $w=0.25$ )

Figure 1: Paint-like compositing based on RYB color model.

\section{Introduction}

Many people have been familiar with subtractive color model based on pigment color compositing since their early childhood. However, the RGB color space is not comprehensible for children due to additive color compositing. In the RGB color space, the resulting mixture color is often different from colors viewer expected. CMYK is a well-known subtractive color space, but its three primal colors are not familiar. Kubelka-Munk model (KM model in short) simulates pigment compositing as well as paint-like appearance by physically-based simulation. However, it is difficult to use KM model because of many simulation parameters.

In order to realize easy to use for paint-like compositing, we present two color compositing methods based on an RYB color model. The RYB color model proposed by Johannes Itten [1973] is widely used in art education, where red, yellow and blue are defined as subtractive primaries. Gossett and Chen [2004] proposed a conversion method from RGB color space to RYB color space based on tri-linear interpolation among three primaries. However, these conversion method is irreversible, i.e., conversion equations between RGB and RYB color models have not been proposed yet.

In this paper, we formulate mathematical and bi-directional conversion equations between RGB and RYB color spaces. We also propose simple color compositing methods to represent lower and highly scattering pigments compositing.

\section{RYB Color Model and Compositing}

We derive conversion equations between RGB, $\left(R_{R G B}, G_{R G B}, B_{R G B}\right)$, and RYB, $\left(R_{R Y B}, Y_{R Y B}, B_{R Y B}\right)$, color spaces. Note that $0 \leq R_{R G B}$, $G_{R G B}, B_{R G B}, R_{R Y B}, Y_{R Y B}, B_{R Y B} \leq 1$.

\subsection{RGB to RYB Conversion}

We introduce two sets of variables, $\left(r_{R G B}, g_{R G B}, b_{R G B}\right)$, and $\left(r_{R Y B}\right.$, $\left.y_{R Y B}, b_{R Y B}\right)$, which are calculated by the following equations.

$$
\begin{aligned}
& \left(r_{R G B}, g_{R G B}, b_{R G B}\right)=\left(R_{R G B}, G_{R G B}, B_{R G B}\right)-\left(I_{w}, I_{w}, I_{w}\right) \\
& \left\{\begin{array}{l}
r_{R Y B}=r_{R G B}-\min \left(r_{R G B}, g_{R G B}\right) \\
y_{R Y B}=\left(g_{R G B}+\min \left(r_{R G B}, g_{R G B}\right)\right) / 2 \\
b_{R Y B}=\left(b_{R G B}+g_{R G B}-\min \left(r_{R G B}, g_{R G B}\right)\right) / 2
\end{array}\right.
\end{aligned}
$$

Here, $I_{w}=\min \left(R_{R G B}, G_{R G B}, B_{R G B}\right)$ is a white component in RGB color space. These variables are normalized as

$$
\left(r_{R Y B}^{\prime}, y_{R Y B}^{\prime}, b_{R Y B}^{\prime}\right)=\left(r_{R Y B} / n, y_{R Y B} / n, b_{R Y B} / n\right)
$$

$$
\text { where } n=\max \left(r_{R Y B}, y_{R Y B}, b_{R Y B}\right) / \max \left(r_{R G B}, g_{R G B}, b_{R G B}\right) \text {. }
$$

Finally, by adding a black component, $I_{b}=\min \left(1-R_{R G B}, 1-\right.$ $\left.G_{R G B}, 1-B_{R G B}\right),\left(R_{R Y B}, Y_{R Y B}, B_{R Y B}\right)$ in RYB color space are obtained by the following equations.

$\left(R_{R Y B}, Y_{R Y B}, B_{R Y B}\right)=\left(r_{R G B}^{\prime}, g_{R G B}^{\prime}, b_{R G B}^{\prime}\right)+\left(I_{b}, I_{b}, I_{b}\right)$

\subsection{RYB to RGB Conversion}

A black component $I_{b}=\min \left(R_{r y b}, Y_{r y b}, B_{r y b}\right)$ is subtracted from each component $R_{R Y B}, Y_{R Y B}, B_{R Y B}$ in RYB color space:

$\left(r_{R Y B}, g_{R Y B}, b_{R Y B}\right)=\left(R_{R Y B}, Y_{R Y B}, B_{R Y B}\right)-\left(I_{b}, I_{b}, I_{b}\right)$

Three variables, $\left(r_{R G B}, g_{R G B}, b_{R G B}\right)$ are obtained as follows:

$$
\left\{\begin{array}{l}
r_{R G B}=r_{R Y B}+y_{R Y B}-\min \left(y_{R Y B}, b_{R Y B}\right) \\
g_{R G B}=y_{R Y B}+2 \min \left(y_{R Y B}, b_{R Y B}\right) \\
b_{R G B}=2\left(b_{R Y B}-\min \left(y_{R Y B}, b_{R Y B}\right)\right)
\end{array}\right.
$$

These variables are normalized as

$$
\left(r_{R G B}^{\prime}, g_{R G B}^{\prime}, b_{R G B}^{\prime}\right)=\left(r_{R Y B} / n, g_{R Y B} / n, b_{R Y B} / n\right)
$$

where, $n=\max \left(r_{R G B}, g_{R G B}, b_{R G B}\right) / \max \left(r_{R Y B}, y_{R Y B}, b_{R Y B}\right)$.

Finally, by adding a white component $I_{w}=\min \left(1-R_{R Y B}, 1-\right.$ $\left.Y_{R Y B}, 1-B_{R Y B}\right),\left(R_{R G B}, G_{R G B}, B_{R G B}\right)$ are obtained as:

$$
\left(R_{R G B}, G_{R G B}, B_{R G B}\right)=\left(r_{R G B}^{\prime}, g_{R G B}^{\prime}, b_{R G B}^{\prime}\right)+\left(I_{w}, I_{w}, I_{w}\right)
$$

\subsection{RYB Color Compositing Methods}

According to our experiments (see detailed supplemental documents), we propose two simple RYB compositing methods to represent lower and highly scattering pigments respectively.

We adopt $\alpha$-compositing for lower scattering pigments (e.g. water color) with a parameter $\alpha$. Compositing color $C_{a}$ is calculated from given foreground color $C_{\text {fore }}$ and background color $C_{b a c k}$ in the RYB color space as follows:

$C_{a}=\alpha C_{\text {fore }}+(1-\alpha) C_{\text {back }}(0 \leq \alpha \leq 1)$

In order to represent highly scattering pigments (e.g. gouache or acrylic), we modify $\alpha$-compositing with a parameter $w$ :

$$
C_{m}=C_{\text {fore }}+w C_{\text {back }}(0 \leq w \leq 1)
$$

\section{Results and Conclusion}

Figure 1 shows stroke rendering results by our compositing methods in the RYB color space. Figure 1 (a) shows a result by the $\alpha$-compositing method to represent lower scattering pigments. Figure 1 (b) shows another result by the modified $\alpha$-compositing method to represent highly scattering pigments. The modified $\alpha-$ compositing method enhanced foreground colors more than the $\alpha$ compositing method.

We formulated conversion between RGB and RYB, and verified that paint-like appearance can be realized easily.

\section{References}

Gosett, N., And Chen, B. 2004. Paint inspired color mixing and compositing for visualization. In Proc. INFOVIS 2004, 113-118.

ITTEN, J. 1973. The Art of Color: the subjective experience and objective rationale of color, New York, Van Nostrand Reinhold.

*\{sugita, toki\}@vcl.jp 